\title{
Parâmetros ecocardiográficos em cães saudáveis tratados com acepromazina, meperidina e sua associação
}

\author{
[Echocardiographic parameters in healthy dogs treated with acepromazine, \\ meperidine and their association] \\ A.C. Reis ${ }^{1}$, K.P. Aptekmann ${ }^{2 *}$, L. Egert ${ }^{1}$, P.S.C. Andrade-Júnior ${ }^{3}$ \\ ${ }^{1}$ Aluno de pós-graduação - Universidade Federal do Espírito Santo - UFES - Alegre, ES \\ ${ }^{2}$ Centro de Ciências Agrárias e Engenharias da UFES - Alegre, ES \\ ${ }^{3}$ Hospital Veterinário - Centro de Ciências Agrárias e Engenharias - UFES - Alegre, ES
}

\begin{abstract}
RESUMO
Objetivou-se avaliar os efeitos da tranquilização com meperidina, acepromazina e de sua associação sobre os parâmetros ecocardiográficos em cães. Foram utilizados 12 cães adultos, da raça Rottweiler, submetidos ao exame ecocardiográfico sem utilização de sedação (controle - TC) e a três protocolos de tratamento, utilizando-se meperidina (TM), acepromazina (TA) e a associação dos medicamentos (TMA). As variáveis foram analisadas pelo teste de Tukey $(\mathrm{P}<0,05)$. Observou-se que as médias obtidas na onda A do fluxo mitral em TA e TMA diminuíram significativamente com relação ao TM, que não diferiu do TC. Houve uma diminuição significativa no valor de movimento anular mitral (MAM) e excursão sistólica do plano anular tricúspide (ESPAT) no TA. Não houve diferença significativa para os valores de fração de encurtamento (FE) entre TA e os demais tratamentos. Entretanto, observou-se que $57,3 \%$ dos cães apresentaram valores de FE abaixo da normalidade. As alterações encontradas podem ser decorrentes dos efeitos hipotensores da acepromazina utilizada de forma isolada. Conclui-se que a meperidina ou sua associação com acepromazina não alteram os parâmetros ecocardiográficos em cães saudáveis e que a acepromazina, utilizada isoladamente, causa alteração nos parâmetros de função sistólica dos cães, não sendo recomendada para a contenção química dos cães submetidos ao ecocardiograma, o que poderia levar à má interpretação do exame.
\end{abstract}

Palavras-chave: cardiologia, tranquilização, neuroleptoanalgesia

\begin{abstract}
The aim of this study was to evaluate the effects of sedation with meperidine, acepromazine and its association on the echocardiographic parameters in dogs. Twelve adult Rottweilers were used and subjected to the echocardiography examination without the use of sedation (control - CT) and subjected to three treatment protocols using meperidine (MT), acepromazine (AT), and the combination of drugs (MAT). Variables were analyzed by Tukey test $(p<0,05)$. The averages obtained in A-wave of mitral inflow in AT and MAT decreased significantly compared to MT, which did not differ from CT. There was a significant decrease in the measurement of mitral annulus motion (MAM) and tricuspid annular plane systolic excursion (TAPSE) at TA. There was no significant difference in shortening fraction (SF) values between TA and other treatments. However, it was observed that $57.3 \%$ of the dogs showed SF values below the normal range for the species. All changes found may be due to the hypotensive effects of acepromazine used in isolation. In conclusion, meperidine or its association with acepromazine does not alter echocardiographic parameters in healthy dogs and acepromazine, used alone, causes changes in the parameters of systolic function and is not recommended for sedation of dogs submitted to echocardiogram, since it could cause a misinterpretation of the exam.
\end{abstract}

Keywords: cardiology, sedation, neuroleptoanalgesic

Recebido em 29 de fevereiro de 2016

Aceito em 9 de julho de 2016

*Autor para correspondência (corresponding author)

E-mail: kapreising@gmail.com 


\section{INTRODUÇÃO}

O exame ecocardiográfico possibilita a visibilização de diversas estruturas do coração, fornecendo informações precisas sobre morfologia, fisiologia, hemodinâmica e função cardíaca, o que permite a avaliação do tamanho de câmaras cardíacas e a identificação de lesões valvulares, massas, defeitos congênitos, estenoses e efusão pericárdica, possibilitando o diagnóstico de diversas doenças cardíacas adquiridas ou congênitas em cães (Chetboul, 2010; Caivano et al., 2012; Gomez et al., 2012).

Movimentos do próprio animal ou movimentos respiratórios durante a realização do exame podem gerar artefatos, dificultando a interpretação do exame ou levando o examinador a uma interpretação errônea dele. Para minimizar esse tipo de artefato, pode-se utilizar a tranquilização do animal (Boon, 2011), tornando-se importante o conhecimento das alterações cardiovasculares promovidas pelos fármacos utilizados, evitando-se, desse modo, a interferência na interpretação do exame (Lopes et al., 2011).

Ao se combinar um tranquilizante com um opioide, consegue-se um efeito conhecido como neuroleptoanalgesia, ocorrendo, assim, sedação e analgesia muito maiores do que quando estes são utilizados isoladamente (Spinosa et al., 2011). Opioides, como a meperidina, e os fenotiazínicos, como a acepromazina, são substâncias amplamente utilizadas na medicina veterinária e possuem poucos efeitos cardiovasculares (Spinosa et al., 2011). Entretanto, a acepromazina pode causar hipotensão e depressão do centro vasomotor (Alvaides et al., 2008; Saponaro et al., 2013; Tranquilli et al., 2014) e a meperidina pode provocar efeito inotrópico negativo (Tranquilli et al., 2014) e cronotrópico positivo (Spinosa et al., 2011; Vettorato e Bacco, 2011).

Considerando-se os efeitos citados acima, que podem influenciar tanto a pré-carga quanto a pós-carga, espera-se encontrar diminuição nos parâmetros de contratilidade cardíaca e alteração nos parâmetros dependentes da pré e pós-carga. Objetivou-se com o presente estudo avaliar as possíveis alterações nos parâmetros ecocardiográficos causadas pela administração de meperidina, acepromazina, e de sua combinação, em cães saudáveis.

\section{MATERIAL E MÉTODOS}

Foram utilizados 12 cães adultos, da raça Rottweiler, seis machos e seis fêmeas, pesando entre 33 e $53 \mathrm{~kg}(43,58 \pm 3,45 \mathrm{~kg})$, com idade de 17 a 61 meses $(38,58 \pm 14,04$ meses $)$, provenientes do canil Rancho Piorra, localizado no município de Marataízes, Espírito Santo. O proprietário do canil foi esclarecido sobre $\mathrm{o}$ estudo e assinou o termo livre e esclarecido, autorizando a participação dos animais no estudo. $\mathrm{O}$ estudo foi aprovado pela Comissão de Ética no Uso de Animais (CEUA-UFES), protocolo 12/2015, seguindo os princípios éticos em experimentação animal, padronizados pelo Colégio Brasileiro de Experimentação Animal.

Para incluir cada animal no grupo experimental, foram realizados exame físico (auscultação cardiopulmonar, aferição da temperatura retal, avaliação dos linfonodos, mucosas, tempo de preenchimento capilar, turgor cutâneo e palpação abdominal), exame eletrocardiográfico, ecocardiográfico e aferição da pressão arterial sistólica sistêmica. Qualquer alteração nesses exames excluiu o animal do estudo. O ecocardiograma (ECO) de triagem foi utilizado para a primeira avaliação como tratamento controle (TC) nos cães considerados aptos para o estudo. Os mesmos animais foram submetidos a mais três avaliações ecocardiográficas, utilizando-se diferentes protocolos de sedação, com intervalo de 15 dias entre cada tratamento.

O tratamento meperidina (TM) foi realizado com a aplicação de meperidina, por via intramuscular (IM), na dose de $2 \mathrm{mg} / \mathrm{kg}$. O tratamento acepromazina (TA) consistiu na aplicação da acepromazina, por via IM, na dose de $0,05 \mathrm{mg} / \mathrm{kg}$. O tratamento acepromazina com meperidina (TMA) foi realizado com a utilização da associação, na mesma seringa, de meperidina, na dose de $2 \mathrm{mg} / \mathrm{kg}$, e acepromazina, na dose de $0,05 \mathrm{mg} / \mathrm{kg}$, ambos por via IM.

Para a administração dos medicamentos, os animais foram contidos em estação. Após 20 minutos, foram posicionados em decúbito lateral direito para a avaliação dos parâmetros ecocardiográficos na janela paraesternal direita. Posteriormente à realização e à gravação de 
todas as imagens obtidas em decúbito direito, os animais foram posicionados no decúbito lateral esquerdo para a realização da avaliação dos parâmetros ecocardiográficos nas janelas apical e cranial esquerdas.

Para a realização do exame ecocardiográfico, utilizou-se aparelho ultrassonográfico (Esaote MylabTM 30VET Gold, Brasil), com transdutor setorial PA240 na frequência de 1 a $4 \mathrm{MHz}$. Foram realizados os cortes longitudinais $\mathrm{e}$ transversais do coração, de acordo com recomendações da literatura (Schober e Fuentes, 2001; Boon, 2011; Pariaut et al., 2012).

$\mathrm{Na}$ janela paraesternal direita, no eixo transversal, foram avaliados os seguintes parâmetros no modo M: espessura do septo interventricular em sístole (SIVs) e diástole (SIVd), diâmetro interno do ventrículo esquerdo em sístole (DIVEs) e diástole (DIVEd), espessura da parede livre do ventrículo esquerdo em sístole (PLVEs) e diástole (PLVEd) e distância do septo ao ponto E (E-septo) da válvula mitral. A fração de ejeção $(\mathrm{FEj})$ e a fração de encurtamento (FE) foram calculadas automaticamente pelo equipamento. No modo B, foram avistados: diâmetro interno de átrio esquerdo (AE) e aorta (Ao). A relação átrio esquerdo/aorta (AE/Ao) foi determinada pelo equipamento. $\mathrm{Na}$ imagem de base cardíaca, foi realizada a avaliação do Doppler espectral da velocidade máxima do fluxo pulmonar (VmáxAp) e do Doppler em cores da artéria pulmonar.

$\mathrm{Na}$ janela paraesternal esquerda, foram obtidas imagens nas janelas apical e cranial. $\mathrm{Na}$ janela apical, foram obtidos os valores de Doppler espectral de velocidade de fluxo mitral - mitral A e mitral E, velocidade máxima do fluxo aórtico (Vmáx-Ao) e tempo de relaxamento isovolumétrico (TRIV), e foi avaliado o Doppler em cores das valvas mitral e aorta. A relação onda E/onda A (E/A) foi determinada pelo equipamento. No modo $\mathrm{M}$, foram obtidos os valores de movimento posterior mitral no septo intraventricular (movimento anular mitral MAM) e excursão sistólica do plano anular tricúspide (ESPAT). Na janela cranial, foram obtidas imagens de Doppler em cores de tricúspide.
Para diminuir a influência do peso na avaliação das medidas ecocardiográficas, os parâmetros que variam com o peso, como a espessura de parede e septo (PLVE e SIV), o tamanho de câmara (DIVE) e os movimentos anulares das válvulas (MAM, ESPAT), foram indexados por área corporal. Assim, calculou-se a área corporal $=10,1 \times$ (peso corporal em gramas $^{2 / 3}$ ) $\times 10^{-4}$.

Foi realizado o teste de Kolmogorov-Smirnov para verificar se os dados possuíam distribuição normal, posteriormente procedeu-se à análise de variância e ao teste de Tukey para determinar as diferenças de médias entre os tratamentos. Utilizou-se um nível de significância de 5\% $(\mathrm{P}<0,05)$. Os valores de SIVs, SIVd, DIVEs, DIVEd, PLVEs, PLVEd, Ao, AE, MAM e ESPAT foram avaliados individualmente por apresentarem variação com o peso do animal e foram comparados com os valores de normalidade para a espécie (Gonçalves et al., 2002). A média dos valores de Doppler espectral (onda $\mathrm{E}$, onda $\mathrm{A}, \mathrm{E} / \mathrm{A}, \mathrm{Vmáx}-\mathrm{Ao}$, Vmáx-Ap, TRIV) e a relação AE/Ao, a E-septo, a FE e a Fej foram comparadas com os padrões de normalidade para a espécie canina em geral (Kirberger et al., 1992; Garncarz, 2007; Boon, 2011), independentemente do peso corporal. Foi realizada análise descritiva do Doppler em cores.

\section{RESULTADOS E DISCUSSÃO}

Todos os cães apresentavam-se dóceis durante o manuseio e a realização dos exames ecocardiográficos, facilitando a obtenção das imagens e resultando em um exame de boa qualidade técnica. Cada exame ecocardiográfico durou entre 15 e 20 minutos. Nenhum dos animais apresentou qualquer intercorrência durante a realização dos protocolos experimentais.

Foram determinados os valores médios e o desvio-padrão dos parâmetros ecocardiográficos nos diferentes tratamentos (Tab. 1). Dentre os parâmetros avaliados, apenas a onda $\mathrm{A}$, a relação E/A, o MAM e o ESPAT apresentaram diferença significativa. Nenhum dos cães apresentou alterações na avaliação de Doppler em cores. 
Reis et al.

Tabela 1. Média \pm desvio-padrão dos parâmetros ecocardiográficos de 12 cães da raça Rottweiler submetidos a diferentes protocolos de sedação

\begin{tabular}{|c|c|c|c|c|}
\hline Parâmetros & $\mathrm{TC}$ & $\mathrm{TM}$ & TA & TMA \\
\hline $\mathrm{FC}(\mathrm{bpm})$ & $101,83^{\mathrm{a}} \pm 21,93$ & $92,50^{\mathrm{a}} \pm 16,29$ & $87,58^{\mathrm{a}} \pm 10,86$ & $90,08^{\mathrm{a}} \pm 17,73$ \\
\hline SIVd (mm) & $11,67^{\mathrm{a}} \pm 1,72$ & $11,33^{\mathrm{a}} \pm 1,79$ & $11,33^{\mathrm{a}} \pm 1,59$ & $10,63^{\mathrm{a}} \pm 1,49$ \\
\hline $\operatorname{SIVd}\left(\mathrm{mm} / \mathrm{m}^{2}\right)$ & $9,35^{\mathrm{a}} \pm 1,35$ & $9,08^{\mathrm{a}} \pm 1,45$ & $9,05^{\mathrm{a}} \pm 1,07$ & $8,49^{\mathrm{a}} \pm 1,04$ \\
\hline DIVEd $(\mathrm{mm})$ & $45,06^{\mathrm{a}} \pm 3,73$ & $45,27^{\mathrm{a}} \pm 4,74$ & $41,52^{a} \pm 5,25$ & $40,78^{a} \pm 2,69$ \\
\hline DIVEd $\left(\mathrm{mm} / \mathrm{m}^{2}\right)$ & $36,13^{\mathrm{a}} \pm 3,43$ & $36,24^{\mathrm{a}} \pm 3,62$ & $33,30^{\mathrm{a}} \pm 4,52$ & $32,68^{a} \pm 2,43$ \\
\hline PLVEd (mm) & $12,78^{a} \pm 2,83$ & $13,60^{\mathrm{a}} \pm 1,64$ & $13,36^{\mathrm{a}} \pm 2,09$ & $12,48^{\mathrm{a}} \pm 2,26$ \\
\hline $\operatorname{PLVEd}\left(\mathrm{mm} / \mathrm{m}^{2}\right)$ & $10,24^{\mathrm{a}} \pm 2,36$ & $10,89^{\mathrm{a}} \pm 1,25$ & $10,71^{\mathrm{a}} \pm 1,74$ & $9,97^{\mathrm{a}} \pm 1,69$ \\
\hline SIVs $(\mathrm{mm})$ & $15,12^{\mathrm{a}} \pm 3,51$ & $14,95^{\mathrm{a}} \pm 3,15$ & $14,57^{\mathrm{a}} \pm 1,80$ & $13,99^{\mathrm{a}} \pm 1,76$ \\
\hline $\mathrm{SIVs} / \mathrm{m} 2$ & $12,08^{\mathrm{a}} \pm 2,71$ & $11,97^{\mathrm{a}} \pm 2,53$ & $11,67^{\mathrm{a}} \pm 1,45$ & $11,19^{\mathrm{a}} \pm 1,29$ \\
\hline PLVEs (mm) & $14,40^{\mathrm{a}} \pm 2,01$ & $15,30^{\mathrm{a}} \pm 2,03$ & $15,13^{\mathrm{a}} \pm 1,92$ & $14,35^{a} \pm 1,58$ \\
\hline PLVEs $\left(\mathrm{mm} / \mathrm{m}^{2}\right)$ & $11,53^{\mathrm{a}} \pm 1,62$ & $12,25^{\mathrm{a}} \pm 1,63$ & $12,17^{\mathrm{a}} \pm 1,89$ & $11,49^{\mathrm{a}} \pm 1,28$ \\
\hline DIVEs (mm) & $29,44^{\mathrm{a}} \pm 4,51$ & $28,64^{\mathrm{a}} \pm 2,95$ & $28,86^{\mathrm{a}} \pm 5,02$ & $26,41^{\mathrm{a}} \pm 3,55$ \\
\hline DIVEs $\left(\mathrm{mm} / \mathrm{m}^{2}\right)$ & $23,59^{a} \pm 3,66$ & $22,95^{\mathrm{a}} \pm 2,49$ & $23,12^{\mathrm{a}} \pm 4,10$ & $21,16^{\mathrm{a}} \pm 2,89$ \\
\hline $\mathrm{AE} / \mathrm{Ao}$ & $0,97^{\mathrm{a}} \pm 0,08$ & $0,96^{\mathrm{a}} \pm 0,09$ & $1,00^{\mathrm{a}} \pm 0,08$ & $1,00^{\mathrm{a}} \pm 0,08$ \\
\hline FEj $(\%)$ & $65,33^{a} \pm 5,87$ & $65,17^{\mathrm{a}} \pm 6,31$ & $60,58^{\mathrm{a}} \pm 8,76$ & $65,33^{\mathrm{a}} \pm 8,73$ \\
\hline $\mathrm{FE}(\%)$ & $36,00^{\mathrm{a}} \pm 4,09$ & $35,08^{\mathrm{a}} \pm 4,36$ & $31,92^{\mathrm{a}} \pm 6,47$ & $35,83^{a} \pm 6,52$ \\
\hline Mitral E (m/s) & $0,84^{\mathrm{a}} \pm 0,10$ & $0,81^{\mathrm{a}} \pm 0,09$ & $0,76^{\mathrm{a}} \pm 0,10$ & $0,76^{\mathrm{a}} \pm 0,10$ \\
\hline Mitral A (m/s) & $0,52^{\mathrm{ab}} \pm 0,11$ & $0,56^{\mathrm{a}} \pm 0,09$ & $0,46^{b} \pm 0,07$ & $0,44^{b} \pm 0,07$ \\
\hline $\mathrm{E} / \mathrm{A}$ & $1,64^{\mathrm{ab}} \pm 0,28$ & $1,48^{\mathrm{a}} \pm 0,14$ & $1,70^{\mathrm{ab}} \pm 0,26$ & $1,74^{\mathrm{b}} \pm 0,21$ \\
\hline Vmáx-Ao (m/s) & $1,16^{\mathrm{a}} \pm 0,20$ & $1,18^{\mathrm{a}} \pm 0,17$ & $1,09^{\mathrm{a}} \pm 0,12$ & $1,02^{\mathrm{a}} \pm 0,17$ \\
\hline Vmáx-Ap (m/s) & $1,08^{\mathrm{a}} \pm 0,11$ & $1,19^{\mathrm{a}} \pm 0,12$ & $1,06^{\mathrm{a}} \pm 0,12$ & $1,07^{\mathrm{a}} \pm 0,14$ \\
\hline TRIV (ms) & $61,83^{a} \pm 6,15$ & $63,10^{\mathrm{a}} \pm 11,00$ & $61,33^{a} \pm 5,07$ & $64,33^{a} \pm 6,27$ \\
\hline E-septo (mm) & $4,06^{\mathrm{a}} \pm 0,96$ & $4,68^{\mathrm{a}} \pm 0,98$ & $4,43^{\mathrm{a}} \pm 0,71$ & $3,83^{\mathrm{a}} \pm 1,34$ \\
\hline MAM (mm) & $10,75^{\mathrm{a}} \pm 0,92$ & $10,20^{\mathrm{ab}} \pm 1,04$ & $9,13^{\mathrm{b}} \pm 1,24$ & $10,26^{\mathrm{ab}} \pm 1,13$ \\
\hline $\operatorname{MAM}\left(\mathrm{mm} / \mathrm{m}^{2}\right)$ & $8,62^{\mathrm{a}} \pm 0,84$ & $8,17^{\mathrm{a}} \pm 0,79$ & $7,31^{b} \pm 0,95$ & $8,23^{\mathrm{ab}} \pm 1,02$ \\
\hline $\operatorname{ESPAT}(\mathrm{mm})$ & $20,87^{a} \pm 2,00$ & $19,48^{\mathrm{a}} \pm 1,98$ & $16,53^{b} \pm 2,53$ & $20,01^{a} \pm 2,46$ \\
\hline $\operatorname{ESPAT}\left(\mathrm{mm} / \mathrm{m}^{2}\right)$ & $16,79^{a} \pm 2,23$ & $15,65^{\mathrm{a}} \pm 1,95$ & $13,24^{\mathrm{b}} \pm 2,01$ & $16,11^{\mathrm{a}} \pm 2,52$ \\
\hline
\end{tabular}

Variáveis com letras diferentes apresentam diferença significativa $(\mathrm{P}<0,05)$.

Legenda: FC - frequência cardíaca; SIVd - septo interventricular em diástole; DIVEd - diâmetro do ventrículo esquerdo em diástole; PLVEd - parede livre do ventrículo esquerdo; SIVs - septo interventricular em sístole; PLVEs parede livre do ventrículo esquerdo em sístole DIVEs - diâmetro do ventrículo esquerdo em sístole; AE/Ao - relação átrio esquerdo/aorta; FEj - fração de ejeção; FE - fração de encurtamento; mitral E - enchimento rápido do ventrículo esquerdo; mitral A - enchimento lento do ventrículo esquerdo; E/A - relação mitral E/mitral A; Vmáx-Ao velocidade máxima do fluxo aórtico; Vmáx-Ap - velocidade máxima do fluxo da artéria pulmonar; TRIV - tempo de relaxamento isovolumétrico; E-septo - distância do septo interventricular ao ponto E; MAM - movimento anular mitral; ESPAT - excursão sistólica do plano anular tricúspide; TC - tratamento controle; TM - tratamento meperidina; TA - tratamento acepromazina; TMA - tratamento acepromazina e meperidina.

Observou-se diferença significativa para onda A do fluxo mitral entre TM e TA, e TM e TMA. Ao se analisar as médias obtidas em cada tratamento, notou-se que os valores obtidos em TA e TMA $(0,46$ e $0,44 \mathrm{~m} / \mathrm{s}$, respectivamente) diminuíram com relação ao TM $(0,56 \mathrm{~m} / \mathrm{s})$, que não diferiu do TC. Não houve diferença significativa nos valores da onda E. Devido à redução nos valores de onda $\mathrm{A}$, a razão entre $\mathrm{E} / \mathrm{A}$ foi alterada significativamente quando comparados os tratamentos TM e TMA. Observando-se as médias obtidas em TM $(1,48 \pm$ $0,14)$ e TMA $(1,74 \pm 0,21)$, percebe-se um pequeno aumento na razão E/A devido à diminuição da velocidade da onda A. Mesmo apresentando diferença significativa entre os tratamentos, todos os cães apresentaram valores normais do fluxo mitral, segundo Boon (2011).

A causa da diminuição na onda $\mathrm{A}$, em TA e TMA, pode ser atribuída à provável hipotenção causada pela acepromazina (Grasso et al., 2015). Com o menor aporte sanguíneo chegando ao coração, a passagem passiva do sangue do átrio esquerdo para o VE (onda E) se mantém constante, restando um menor volume para a 
passagem ativa (onda A), o que provoca uma diminuição na velociade máxima da onda $\mathrm{A}$.

Observou-se diminuição significativa no valor de MAM, em TA, com relação ao valor obtido no TC. Na avaliação do ESPAT, foi verificada diferença significativa entre TA e os demais tratamentos, sendo observada diminuição nos valores. Ambos apresentaram alteração significativa em TA, sugerindo possível diminuição da contratilidade longitudinal dos ventrículos esquerdo e direito quando utilizada a acepromazina isoladamente.

Apesar das alterações encontradas em parâmetros de avaliação da função sistólica (MAM e TAPSE), não houve diferença significativa para os valores da FE entre TA e os demais tratamentos. Entretanto, analisando-se os valores individuais e a média da FE em TA $(31,9$ $\pm 6,5 \%$ ), observou-se que 57,3\% (7/12) dos cães apresentaram valores abaixo da normalidade para a espécie (33,3 a 45,9\%), segundo Boon (2011).

Estes achados sugerem prejuízo nos parâmetros de função sistólica quando a acepromazina foi utilizada isoladamente, podendo ser consequência da hipotensão causada por esse fármaco (Grasso et al., 2015). A hipotensão provoca diminuição do volume sanguíneo que chega ao coração, levando ao menor estiramento das fibras do miocárdio e à diminuição da força de contração, segundo a lei de Frank-Starling (Reece, 2006).

Embora a diminuição da FE fosse esperada nos cães submetidos ao TM, devido ao efeito inotrópico negativo da meperidina (Tranquilli et al., 2014), comprovado experimentalmente em ratos (Zhang et al., 2003), não houve diferença significativa com o TC, e o valor médio apresentou-se dentro dos limites de normalidade para a espécie.

Não houve diferença significativa na FC entre nenhum tratamento. Porém, em outro estudo com cães saudáveis, foi observado que a medetomidina, tanto associada com a acepromazina quanto sozinha, produziu redução significativa da FC e do débito cardíaco, quando comparada com a acepromazina sozinha. A acepromazina diminuiu a pós-carga do $\mathrm{VE}$, enquanto a medetomidina aumentou a pós-carga do ventrículo direito e a pós-carga sistêmica foi levemente diminuída. Quando utilizadas em conjunto, foi possível atenuar o desarranjo da pós-carga dos ventrículos esquerdo e direito produzido pela acepromazina e medetomidina, respectivamente (Saponaro et al., 2013).

Como limitações deste estudo, não foi possível realizar exames de sangue prévios à avaliação ecocardiográfica, porém, por serem animais de competição, todos realizavam avaliações veterinárias periódicas e exames de sangue frequentes que garantiam um bom estado de saúde deles. Na avaliação ecocardiográfica, apesar de o método de Simpson ser o mais recomendado para a avaliação da fração de ejeção, as imagens não ficaram satisfatórias para conter a totalidade do contorno luminal do ventrículo esquerdo, o que resultaria em valores não fidedignos. Para uma avaliação mais completa da função sistólica, os métodos de Strain e Strain Rate poderiam ser utilizados, porém, neste estudo, foi uma limitação do equipamento. Apesar de os parâmetros ecocardiográficos utilizados neste estudo serem subjetivos nas avaliações sistólica e diastólica, eles são utilizados rotineiramente para a avaliação ecocardiográfica de cães, devendo ser cuidadosamente interpretados em animais tranquilizados

\section{CONCLUSÕES}

Em cães saudáveis, a meperidina ou sua associação com acepromazina não alteram os parâmetros ecocardiográficos, podendo ser indicadas na contenção química dos cães para a realização do exame ecocardiográfico. A acepromazina, utilizada isoladamente, causa alteração nos parâmetros de função sistólica dos cães, não sendo recomendada para a contenção química dos animais submetidos ao exame, pois, mesmo que essas alterações sejam decorrentes da hipotensão causada por esse fármaco, elas poderiam levar à má interpretação do exame. 


\section{REFERÊNCIAS}

ALVAIDES， R.K.; TEIXEIRA NETO, F.J.; AGUIAR, A.J.A. et al. Sedative and cardiorespiratory effects of acepromazine or atropine given before dexmedetomidine in dogs. Vet. Rec., v.162, p.852-856, 2008.

BOON, J.A. (Ed.). Veterinary echocardiography. IOWA: John Wiley \& Sons, 2011. 632p.

CAIVANO, D.; BIRETTONI, F.; FRUGANTI, A. et al. Transthoracic echocardiographicallyguided interventional cardiac procedures in the dog. J. Vet. Cardiol., v.14, p.431-444, 2012.

CHETBOUL, V. Advanced techniques in echocardiography in small animals. Vet. Clin. N. Am. Small Anim. Pract., v.40, p.529-543, 2010.

GARNCARZ, M.A. Echocardiographic evaluation of diastolic parameters in dogs with dilated cardiomyopathy. Pol. J. Vet. Sci., v.10, p.207-215, 2007.

GOMEZ, A.; DEL PALACIO, J.F.; LATORRE, $\mathrm{R}$. et al. Plastinated heart slices aid echocardiographic interpretation in the dog. Vet. Radiol. Ultrasound, v.53, p.197-203, 2012.

GRASSO, S.C.; KO, J.C.; WEIL, A.B. et al. Hemodynamic influence of acepromazine or dexmedetomidine premedication in isofluraneanesthetized dogs. J. Am. Vet. Med. Assoc., v.246, p.754-764, 2015.

KIRBERGER, R.M.; BLANDVANDENBERG, P.; GRIMBEEK, R.J. Doppler echocardiography in the normal dog: part 2. factors influencing blood-flow velocities and a comparison between left and right heart blood-flow. Vet. Radiol. Ultrasound, v.33, p.380-386, 1992.

LOPES, B.F.; TAFFAREL, M.O.; FEITOSA, M.L. et al. Hepatic quantitative radiography in dogs with acepromazine. Cienc. Rural, v.41, p.137-142, 2011.
PARIAUT, R.; SAELINGER, C.; STRICKLAND, K.N. et al. Tricuspid annular plane systolic excursion (TAPSE) in dogs: reference values and impact of pulmonary hypertension. J. Vet. Intern. Med., v.26, p.11481154, 2012.

REECE, W.O. (Ed.). Dukes: fisiologia dos animais doméstios. Rio de Janeiro: Guanabara Koogan, 2006. 926p.

SAPONARO, V.; CROVACE, A.; DE MARZO, L. et al. Echocardiographic evaluation of the cardiovascular effects of medetomidine, acepromazine and their combination in healthy dogs. Res. Vet. Sci., v.95, p.687-692, 2013.

SCHOBER, K.E.; FUENTES, V.L. Mitral annulus motion as determined by M-mode echocardiography in normal dogs and dogs with cardiac disease. Vet. Radiol. Ultrasound, v.42, p.52-61, 2001.

SPINOSA, H.S.; GÓRNIAK, S.L.; BERNARDI, M.M. (Eds.). Farmacologia aplicada à medicina veterinária. Rio de Janeiro: Guanabara Koogan, 2011. 824p.

TRANQUILLI, W.J.; THURMON， J.C.; GRIMM, K.A. (Eds.). Anestesiologia e analgesia veterinária. São Paulo: Lumb \& Jones, 2014. $1192 \mathrm{p}$.

VETTORATO, E.; BACCO, S. A comparison of the sedative and analgesic properties of pethidine (meperidine) and butorphanol in dogs. J. Small. Anim. Pract., v.52, p.425-431, 2011.

ZHANG, X.; CAO, C.; WANG, L. et al. Negative inotropic effect of meperidine in rat ventricular muscle and the underlying mechanism. Sheng Li Xue Bao, v.55, p.197-200, 2003. 